\title{
Shear induced formation of carbon and boron nitride nano-scrolls
}

\author{
Xianjue Chen, Ramiz A. Boulos, John F. Dobson and Colin L. Raston*
}

\section{$(($ Dedication----optional $))$}

Two-dimensional graphene has attracted the attention of the wider scientific community because of its extraordinary properties and potential applications. ${ }^{[1,2]}$ Since the original 'scotch tape' exfoliation of graphite flakes into graphene sheets, ${ }^{[3]}$ a large number of synthesis methods have been developed for facile and scalable production of the material with high purity and quality. ${ }^{[4-8]}$ An inherent problem in taking graphene sheets into applications is the instability of the 2D sheets. Even though they are stable when dispersed in certain organic solvents or water after incorporating functionalised groups, ${ }^{[4,5]}$ their integrity can be lost when the nature of the dispersing medium changes. ${ }^{[9]}$ This arises from their high surface energy which tends to result in (a) spontaneous heterogeneous decoration with alien material, (b) restacking or folding, or (c) self-scrolling in affording another type of graphene which can have even more remarkable properties than planar graphene. ${ }^{[10,11]}$ Indeed, these so-called carbon nano-scrolls (CNSs) are predicted to have distinct properties akin to both graphene sheets and carbon nanotubes, ${ }^{[12]}$ with applications in chemical doping, ${ }^{[13]}$ hydrogen storage, ${ }^{[14-16]}$ battery technology, ${ }^{[17]}$ and nano-mechanical devices. ${ }^{[18]}$ An impediment to developing these, however, is being able to access significant quantities of the material with control over their morphology, including their compactness.

Several methods of fabricating CNSs have been reported which include the use of high energy arc-discharge ${ }^{[19]}$ and ball milling, ${ }^{[20]}$ but they are formed in very low yield and are loaded with impurities, with inefficient and high energy usage, and limited control of their morphology. High-quality CNSs can be generated from graphene on $\mathrm{SiO}_{2} / \mathrm{Si}$ substrates in the presence of isopropyl alcohol, ${ }^{[12]}$ but this method will be inherently difficult to scale-up. Alkali metal intercalation of graphite followed by sonication affords some CNSs,

[*] X. Chen, Dr. R. A. Boulos, Prof. C. L. Raston Centre for Strategic Nano-Fabrication, School of Chemistry and Biochemistry, The University of Western Australia, WA 6009 (Australia)

Fax: +61864888683

E-mail: colin.raston@uwa.edu.au

Prof. J. F. Dobson

Queensland Micro- and Nano-technology Centre, Griffith University, Nathan, Queensland 4111 (Australia).

Fax: +61737357656

E-mail: j.dobson@griffith.edu.au

[**] We gratefully acknowledge the Australian Research Council, and the China Scholarship Council for support of this research, and helpful discussions with Prof Martin Saunders. TEM and SEM work was carried out in the Centre for Microscopy, Characterisation and Analysis (The University of Western Australia).

Supporting information for this article is available on the WWW under http://www.angewandte.org or from the author. along with graphene sheets, ${ }^{[21,22]}$ but they have defects and again there is little control over their morphology. The same problems are encountered in fabricating CNSs in liquid nitrogen involving a microwave spark assistance process. ${ }^{[23]}$

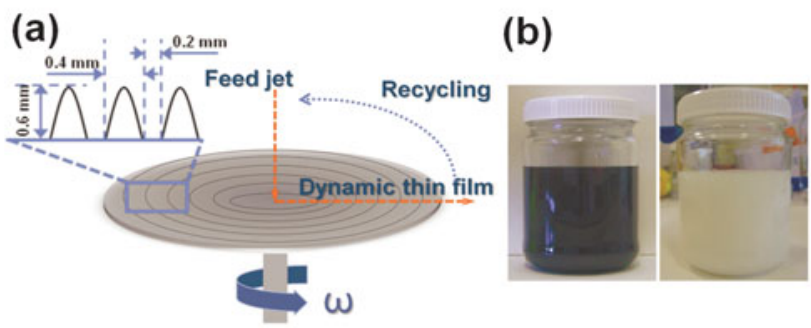

Figure 1. Schematic of the spinning disc processor $(20 \mathrm{~cm}$ diameter stainless steel disc operating at $2500 \mathrm{rpm}$ ). (b) Photographs of 200 $\mathrm{mL}$ suspensions of processed graphite (left) and $h$-boron nitride (right) flakes in NMP.

Herein we develop the use of shear forces in dynamic thin films generated in a spinning disc processor (SDP) ${ }^{[24]}$ to exfoliate pristine graphite flakes, and flakes of the related laminar material $h$-BN, with simultaneous scroll formation, noting there has been no reports of scrolls of this material. A schematic of the SDP is shown in Figure 1a, with further details available (S1, ESI). N-methyl-2pyrrolidone (NMP) was the solvent of choice for the processing, on the grounds that it has a similar surface tension relative to graphene and $\mathrm{BN}$, and can stabilise exfoliated material towards restacking. ${ }^{[5]}$ This work relates to the use of a vortex fluidic device to exfoliate the same materials into $2 \mathrm{D}$ sheets. ${ }^{[8]}$

In a typical experiment, a jet feed of graphite or BN flakes suspensed in NMP was directed close to the centre of a $20 \mathrm{~cm}$ disc rotating at $2500 \mathrm{rpm}$, resulting in a dynamic thin film with high surface area-to-volume ratio, ${ }^{[24]}$ as a continuous flow process, with a $200 \mathrm{~mL}$ solution constantly recycled over 20 hours using a flow rate of $\sim 60 \mathrm{~mL} / \mathrm{min}$. Photographs of the resulting NMP suspensions derived from graphite and BN are shown in Figure 1b. Recycling of the solution overcomes the short residence time of a finite volume of liquid on the rapidly rotating disc which is typically less than a second. SDPs have been used in the 'bottom up' fabrication of nanomaterials, controlling chemical reactivity and selectivity, and probing the structure of self organised systems. ${ }^{[25-27]}$ The latter relates to the present work, but rather than disassembling nanomaterial for spontaneous reassembly post shearing, the 'top-down' exfoliating and scroll formation from micron sized particles of graphite $(7-10 \mu \mathrm{m})$ or $\mathrm{BN}(\sim 1 \mu \mathrm{m})$ is irreversible.

Transmission electron microscopy (TEM) images of CNSs are shown in Figure 2a and 2b. Overall the scrolls are irregular in shape with straight sections connected by bends, and are formed in $\leq 1 \%$ yield. The bends may arise from the impact of the high velocity liquid when it leaves the rapidly rotating disc and strikes the 
collecting walls and/or the way the scrolls form on the surface of the graphite flakes under the intense shear. The CNSs vary considerably in length and diameter, as expected from the different sizes of the graphite flakes in the starting material, although tearing of graphene sheets during the exfoliation/scrolling process cannot be ruled out.

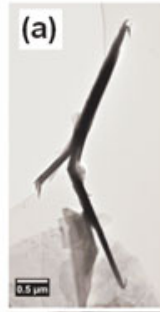

(e)

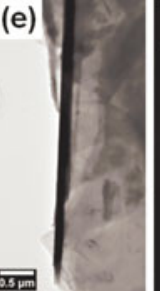

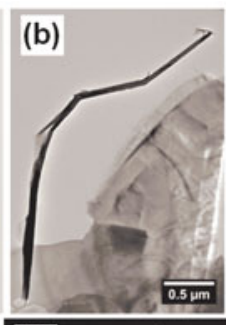

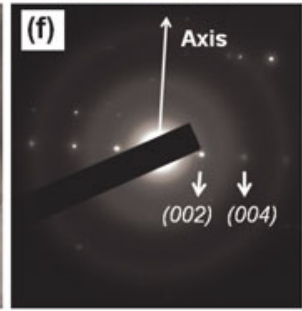

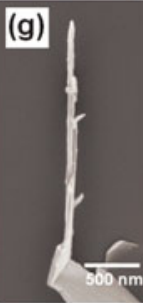

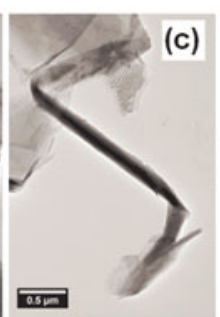

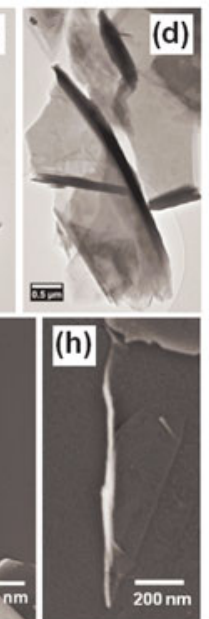

Figure 2. (a) and (b) TEM images of carbon nano-scrolls (CNSs). (c) -(e) TEM images of partial CNSs. (f) Selected area electron diffraction (SAED) pattern of the CNS in (e). (g) and (h) SEM images of CNSs.

A large number of partial CNSs are present, as highlighted by the TEM images in Figures $2 \mathrm{c}$ and 2d, and most of these are on the edge of the graphite flakes, which has mechanistic implications for the scrolling process (see below). They presumably arise from the irregular morphology of the graphite flakes under varying localised microfluidic conditions. Under ideal constant shear, the energy to roll up graphene sheets is expected to rise rapidly as the number of layers of graphene in a CNS increases. ${ }^{[28]}$ A partially rolled-up CNS is shown in Figure 2e, with the selected area electron diffraction (SAED) pattern in Figure 2f, which is similar to that of carbon nanotubes. ${ }^{[29]}$ The pattern can be indexed according to the reciprocal lattice of graphite, with the (002) and (004) spots arising from the bending of the layers in the scroll into cylindrical shapes, which then allows the direction of the axis of the scroll in the diffraction pattern to be determined as perpendicular to the $c$ axis. Scanning electron microscopy (SEM) was also used to characterize the CNSs, Figure $2 \mathrm{~g}$.

$h$-BN can be similarly converted to scrolls in NMP, although now the yield is much higher at $\sim 5 \%$. Given that graphite and BN have similar interlayer binding energy, ${ }^{[30]}$ the higher yield must arise from the use of smaller average particle size particles of flakes, $\sim 1$ $\mu \mathrm{m}$ diameter, compared to $\sim 7-10 \mu \mathrm{m}$ for graphite, with the total energy between sheets lower for a smaller area undergoing exfoliation and scroll formation. ${ }^{[28]}$ TEM images of BN scrolls are shown in Figure 3a, most of them being multi-layered with lengths consistent with the diameter of the precursor flakes. The inset in Figure $3 \mathrm{~b}$ shows the SAED pattern of the scroll, which is similar to that of CNSs. SEM images of BN scrolls are shown in Figure $3 \mathrm{c}$ and 3d. High resolution transmission electron microscopy (HRTEM) for the area highlighted in Figure $3 \mathrm{e}$ show several step-like rolling edges along the scroll, Figure $3 \mathrm{f}$, and that the scroll is tightly rolled up without an internal void. The HRTEM image in Figure $3 \mathrm{~g}$ shows another BN scroll with a hollow core tube-like structure, and is reminiscent of multi-wall $\mathrm{BN}$ tubes. The inside void is $2 \mathrm{~nm}$ in diameter with the distance between successive turns in the scroll at the van der Waals limit, $\sim 0.33 \mathrm{~nm}$. Difference in the structure of the
BN scrolls may be a consequence of the non-uniform nature of the microfluidic conditions on the surface of the flakes under shear.
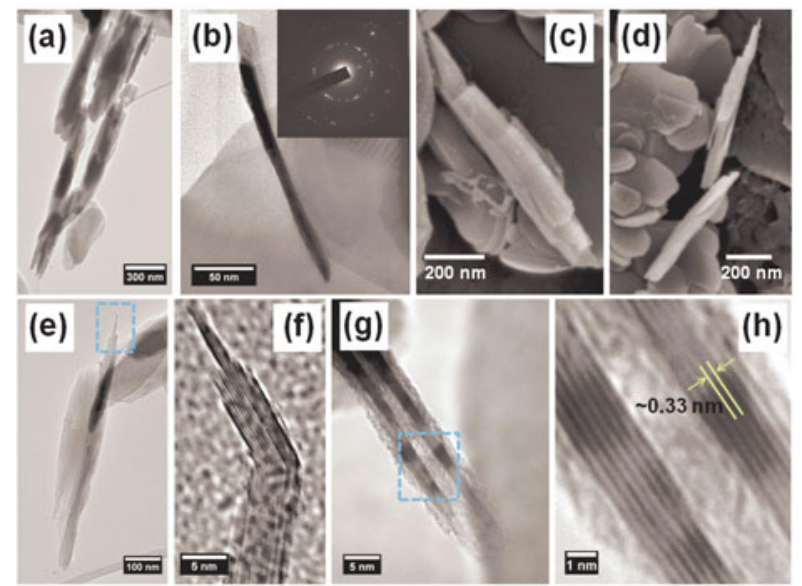

(h)

Figure 3. BN scrolls: (a) and (b) TEM images with a SAED pattern inset. (c) and (d) SEM images. (e) TEM image with an HRTEM image of the area indicated in (f). (g) and (h) HRTEM images.

The potential mechanism of exfoliation with scroll formation using SDP is shown in Figure 4. Suspensions of flakes of graphite or $\mathrm{BN}$ directed onto the spinning disc are immediately accelerated by the centrifugal effect, resulting in a dynamic thin film typically $\leq$ $200 \mu \mathrm{m}$ thick. ${ }^{[31]}$ The flakes will move in the thin film with periodic contact with the surface of the disc, where they will experience shear forces arising from the viscous drag of the liquid on the surface of the disc. The shear forces and indeed collision frequency of the flakes with the surface of the disc will be enhanced using a disc with periodically arranged grooves, as in the present study, Figure 1(a). The shear forces provide the energy for exfoliation, with three different subsequent events possible, as concluded from screening a large number of samples using SEM, Figure 4. Lift from the shear force on the upper layers of the flakes may facilitate exfoliation of the bottom layers with different degrees of folding, Figure $4 \mathrm{a}$ and $4 \mathrm{~b}$. Another scenario is that the edge of the flakes can curve to eventually form scrolls, Figure $4 \mathrm{c}$. The exfoliation results in an apparent reduction in the thickness of the flakes (Figure S2, ESI).

The formation of scrolls versus folding will depend on the nature of the material, the orientation of the flake, the shear intensity, and the number of layers exfoliated at the same time. Molecular modelling in vacuo of a single sheet of graphene using the starting geometries in Figure 5(a) favours spontaneous scrolling once the sheet is curved to the extent that the edge of the sheet makes sufficient contact with its inner surface. For the analysis that follows we introduce the arc length, $s$, and radius, $r$, of the curved (rolledup) part of the sheet as viewed in cross section (see Fig 5b). The scrolling occurs when rolled arc length $s$ of the sheet is $>2 \pi \mathrm{r}(\mathrm{r}=$ $1.115 \mathrm{~nm}, s>6.0 \mathrm{~nm}$ ) (S3 ESI). Below this value the minimized structure is almost a flat sheet, although there is a stable intermediate structure shown in Figure 5(c) and (d). Figure 5(e) shows the energies of a graphene sheet and scroll after energy minimisation relative to that of a flat sheet $(s=0)$. As the scrolling process is initiated, the internal energy (red line) of the graphene sheet gradually increases as bond stretching and bond bending reduces the overlap between the $\mathrm{sp}^{2}$ hybridized carbon atoms contributing to the $\pi$ bonding. As the rolled arc length $s$ approaches the circumference of the scroll, the internal energy of the scroll increases significantly, and at a critical point it is counter balanced by the energy of the non-bonding interactions (green line) between 
the scrolls, Figure 5(e) $(s=5.6 \mathrm{~nm})$. Here the total energy (blue line) is sufficient to stabilize the sheet in this form, but beyond this point the $\pi-\pi$ interactions favour scrolling, with a final radius of $0.615 \mathrm{~nm}$, and an intra-sheet distance of $0.388 \mathrm{~nm}$, which compares with 0.33 $\mathrm{nm}$ experimentally, Figure $3 \mathrm{~h}$. Previous studies have shown that van der Waals interactions between sheets dominate the scrolling, ${ }^{[13,28]}$ and scrolling a graphene sheet on a graphite flake beyond the above intermediate structure has to overcome such interactions.

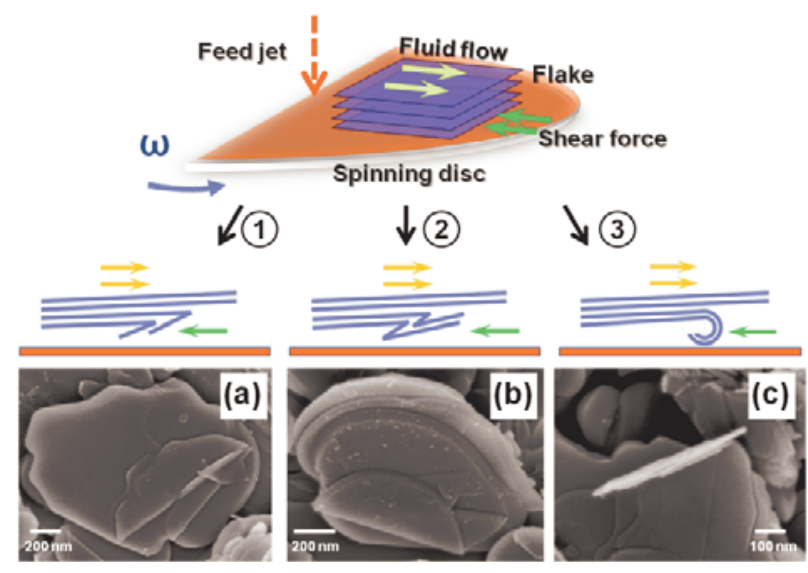

Figure 4. Proposed mechanism of exfoliation and scrolling of graphite and BN flakes using SDP, with supporting SEM images.

There is a relationship between the rolled arc length $s$ and the time taken for the energy of the scrolls to converge (S3 ESI) with the time gradually increasing up to $s=s_{0}<2 \pi \mathrm{r}$, and then decreasing for further scrolling, suggesting that the intermediate geometry falls in a potential energy well. Sheets that scroll completely have concomitant scrolling at the other end of the sheet, Figure 5(c) and (d). Also noteworthy is that the length $\mathrm{L}$ of the minimised scroll, for $s=6.0 \mathrm{~nm}$, is the same as the length of the flat sheet whereas the intermediate structure, with $s=5.6 \mathrm{~nm}$, has a larger length L', Figure $5(\mathrm{~d})$. This is associated with the $\mathrm{C}-\mathrm{C}$ bond length $\left(\mathrm{b}_{2}\right.$, Figure 5(c)) parallel to the direction of the scroll changing from $1.399 \AA$ to $1.401 \AA$ for the sheet with $s=6.0 \mathrm{~nm}$, and to $1.481 \AA$ for a sheet with $s=5.6 \mathrm{~nm}$. Other $\mathrm{C}-\mathrm{C}$ bond lengths $\left(\mathrm{b}_{1}\right)$ change from $1.399 \AA$ for a graphene sheet to $1.367 \AA$ for the sheet with $s=5.6 \mathrm{~nm}$ and no change for the sheet with $s=6.0 \mathrm{~nm}$. Similar changes in C-C bond lengths have been reported for carbon nanotubes for different orientations of the six-membered rings along the tube. ${ }^{[32-35]}$

An analytic continuum model provides an understanding of the scroll formation on a graphite flake. Once the leading edge of the scroll makes contact with the opposite surface of the sheet, the subsequent rolling process depends on a competition between (usually negative) graphene-graphene surface (contact) energy and a positive bending energy. If a scroll of length $\mathrm{L}$ rolls up slightly, creating an additional arc length $\Delta s$ of curved surface, an additional area of overlap $\Delta \mathrm{A}=\mathrm{L} \Delta \mathrm{s}$ is created. This causes a free energy change $-\sigma \mathrm{L} \Delta \mathrm{s}$ where $\sigma$ is the contact free energy per unit area in the presence of the liquid, defined so that it is positive. Simultaneously, on dimensional grounds the bending of a previously flat portion of the sheet to feed the scroll must cost a positive bending energy $\varepsilon r^{-2} \mathrm{~L} \Delta \mathrm{s}$, where $\mathrm{r}$ is the radius of the scroll. Here $\varepsilon$ is a characteristic positive energy related to bond bending which can be derived from elasticity theory, thereby providing an expression for $\varepsilon$ in terms of the elastic constants of the sheet. The value of $\varepsilon$ depends on the direction of scrolling relative to the crystallographic axes of the sheet, and is roughly proportional to the number of layers that are being rolled simultaneously.

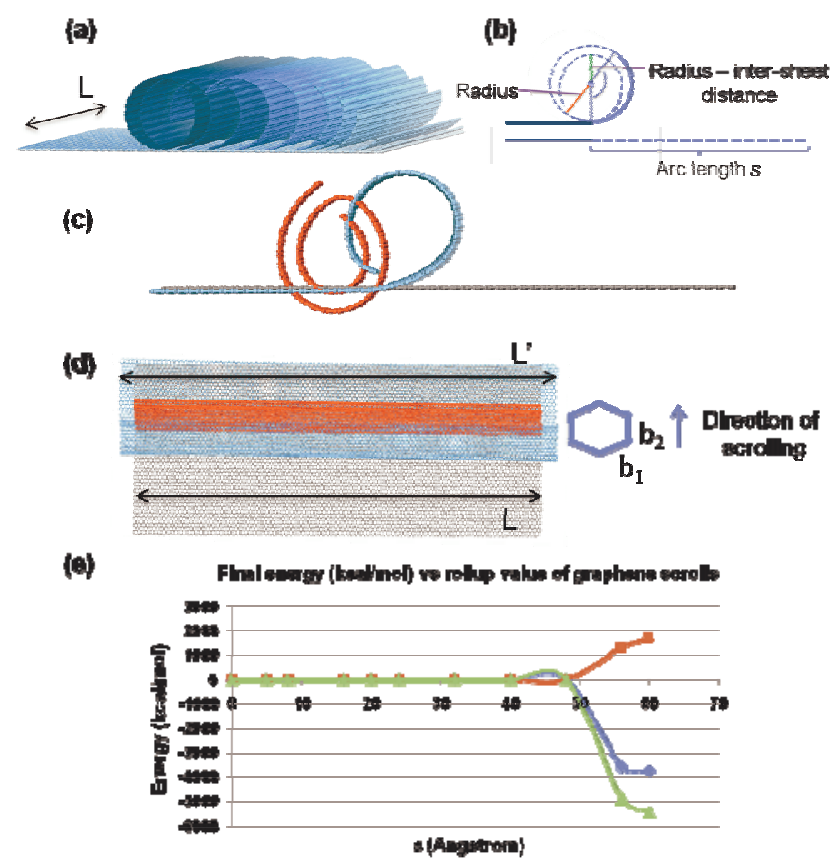

Figure 5. (a) Superimposed starting geometries of a graphene sheet en route to a scroll. (b) Side view and (c) top view of the final geometry for a scroll (red) with that of the intermediate geometry at the threshold between relaxing to a flat sheet and forming a scroll (blue) and a graphene sheet (grey). (d) Final energy versus roll-up arc length $s$, with the blue line representing total energy, the red the internal energy, and the green line the non-bonding energy $(\mathrm{kcal} / \mathrm{mol})$.

The rolling up process for a free scroll in the liquid will occur spontaneously if the net free energy increment of rolling is negative: i.e. if $-\sigma L \Delta s+\varepsilon r^{-2} L \Delta s<0$. Thus rolling is spontaneous for a large enough radius $\mathrm{r}$,

$$
\mathrm{r}>\mathrm{R}_{\text {spont }} \equiv(\varepsilon / \sigma)^{1 / 2}
$$

In water, the graphene-graphene or BN-BN sticking energy $\sigma$ is large and any initiated scrolls will spontaneously roll up tightly according to the above equation (i.e. $\mathrm{R}_{\text {spont }}$ is small). In a solvent such as NMP, $\sigma$ is small, so only large-radius graphene or BN scrolls will form spontaneously once initiated (i.e. $\mathrm{R}_{\text {spont }}$ is large). Since the bending energy $\varepsilon$ is roughly proportional to thickness of a sheet, multilayer sheets will form scrolls of larger radius than singlelayer sheets.

In general the initial stage of curling, before the leading edge makes contact with the parent sheet, involves the positive bending energy only, and this must be supplied externally, ie. from the fluid flow, as depicted Figure 4 process 3. After pinning on the disc, the upper sheets are pushed to the right while the scroll remains stuck on the substrate. If the upper nearest sheet remains flat and does not make contact with the disc, and the radius of curvature of the peeled section is uniform, then the net incremental energy in the curling process initiated via fluid flow becomes

$$
\Delta \mathrm{E}=\left(\sigma_{\mathrm{U}}-\sigma\right) \mathrm{L} \Delta \mathrm{s}+\varepsilon \mathrm{r}^{-2} \mathrm{~L} \Delta \mathrm{s} \approx \varepsilon \mathrm{r}^{-2} \mathrm{~L} \Delta \mathrm{s}>0 .
$$

Here the additional negative energy of sticking inside the scroll is approximately cancelled by the energy cost $\sigma_{\mathrm{U}} \mathrm{L} \Delta \mathrm{s}$ to peel the scrolling layer(s) off the upper sheets, characterized by a surface energy $\sigma_{U}$. This cancellation occurs presumably because $\sigma_{U}$ is quite close to the surface energy for the scroll to stick to itself, $\sigma_{U} \approx \sigma$. 
Thus not just initiation, but the entire rolling-up process in this model costs a positive bending energy, which must be supplied by the shear in the thin film.

We have established that pristine graphite and $h$-BN flakes can form scrolls in NMP in the presence of intense shear generated in a spinning disc processor (SDP) under ambient conditions. The yield of scrolls from $h$-BN flakes is much higher than that from graphite flakes, which relates directly to the difference in the particle size of the precursor material. Potentially, the yield of scrolls can be increased with longer processing time and high spinning rates, and use of SDP is suitable for scaling up. The mechanism of formation of the scrolls is understood through a combination of experimental results, molecular simulations for a single graphene sheet, and an analytic continuum model for the scrolling on the graphite flake. The results establish a basis for understanding the scrolling process and gaining access to nano-materials from the 'top down' processing of laminar compounds with inherently weak interactions between layers, and provide a basis for the future applications of nano-scrolls.

\section{Experimental Section}

Graphite flakes 7-10 microns in diameter were used as received from Alfa Aesar. $h$-BN powder flakes $\sim 1 \mu \mathrm{m}$ in diameter, and N-methyl-2pyrrolidone (NMP) were used as received from Sigma Aldrich. Graphite and BN flakes were suspended in $200 \mathrm{~mL}$ of NMP in targeting 1 and $2 \mathrm{mg} / \mathrm{mL}$ concentrations respectively of suspended material following SDP experiments. The suspensions were directly used for SDP experiments under ambient conditions, without any pretreatments. The disc was constructed of stainless steel, and was cleaned prior to use using distilled water then ethanol. Silicone tubes were used to connect the suspension in a glass vial with the input and output of SDP, respectively, for the recycling. The spinning disc, manufactured by AC Tech Company, $20 \mathrm{~cm}$ in diameter, with a rough surface (concentric grooves $0.6 \mathrm{~mm}$ high, $0.4 \mathrm{~mm}$ wide, and $0.2 \mathrm{~mm}$ spacing) was used in the experiments. The feeding rate was adjusted by a Masterflex ${ }^{\circledR}$ controller from Colo-Parmer Instrument Company and set at $60 \mathrm{~mL} / \mathrm{min}$. The spinning rate was fixed at $2500 \mathrm{rpm}$ and the processing time was 20 hours. A magnetic stirrer was used during the entire process to ensure that the flakes of the two compounds were dispersed homogeneously in the reservoir of solution prior to delivery to the surface of the disc.

The as-treated suspensions were dropped onto 200 mesh holey carbon $\mathrm{Cu}$ grids for TEM characterization, and the micrographs were recorded using JEOL 2100 instruments operating at $120 \mathrm{keV}$. SEM studies were carried out using Zeiss 1555 VP-FESEM with samples coated with $3 \mathrm{~nm}$ Pt films.

Materials studio V5.5.3 was used for the molecular modelling of the CNSs. A script using the programming language 'Frink' was used to construct a graphene sheet $24 \times 10 \mathrm{~nm}$ which was then used to produce CNSs with a radius of $1.115 \mathrm{~nm}$ and different rolled-up arc lengths $s$. The Discover module in Materials Studio V5.5.3 was used for the energy minimizations of the graphene sheet and the carbon scrolls. The PCFF forcefield was used with the conjugate gradient method and the Polak-Ribiere algorithm with a convergence criterion of $10 \mathrm{kcal} / \mathrm{mol} / \AA$ and $1,000,000$ steps.

Received: ((will be filled in by the editorial staff))

Published online on ((will be filled in by the editorial staff))
[1] R. S. Ruoff, Nature 2012, 483, S42.

[2] N. Savage, Nature 2012, 483, S30.

[3] K. S. Novoselov, A. K. Geim, S. V. Morozov, D. Jiang, S. V. Dubonos, I. V. Grigorieva, A. A. Firsov, Science 2004, 306, 666-669.

[4] D. Li, M. B. Muller, S. Gilje, R. B. Kaner, G. G. Wallace, Nat. Nanotechnol. 2008, 3, 101-105.

[5] Y. Hernandez, V. Nicolosi, M. Lotya, F. M. Blighe, Z. Sun, S. De, I. T. McGovern, B. Holland, M. Byrne, Y. K. Gun'ko, J. J. Boland, P. Niraj, G. Duesberg, S. Krishnamurthy, R. Goodhue, J. Hutchison, V. Scardaci, A. C. Ferrari, J. N. Coleman, Nat. Nanotechnol. 2008, 3, 563-568.

[6] X. Li, G. Zhang, X. Bai, X. Sun, X. Wang, E. Wang, H. Dai, Nat. Nanotechnol. 2008, 3, 538-542.

[7] V. C. Tung, M. J. Allen, Y. Yang, R. B. Kaner, Nat. Nanotechnol. 2009, 4, 25-29.

[8] X. Chen, J. F. Dobson, C. L. Raston, Chem. Commun. 2012, 48, 37033705.

[9] R. L. D. Whitby, A. Korobeinyk, K. V. Glevatska, Carbon 2011, 49, 718-736.

[10] D. A. Dikin, S. Stankovich, E. J. Zimney, R. D. Piner, G. H. B. Dommett, G. Evmenenko, S. T. Nguyen, R. S. Ruoff, Nature 2007, 448, 457-460.

[11] X. Ma, M. R. Zachariah, C. D. Zangmeister. Nano Lett. 2012, 12, 486489.

[12] X. Xie, L. Ju, X. Feng, Y. Sun, R. Zhou, K. Liu, S. Fan, Q. Li, K. Jiang, Nano Lett. 2009, 9, 2565-2570.

[13] D. Xia, Q. Xue, J. Xie, H. Chen, C. Lv, F. Besenbacher, M. Dong, Small 2010, 6, 2010-2019.

[14] G. Mpourmpakis, E. Tylianakis, G. E. Froudakis, Nano Lett. 2007, 7, 1893-1897.

[15] S. F. Braga, V. R. Coluci, R. H. Baughman, D. S. Galvao, Chem Phys. Lett. 2007, 441, 78-82.

[16] V. R. Coluci, S. F. Braga, R. H. Baughman, D. S. Galvao, Phys. Rev. 2007, 75, 125404

[17] E. Yoo, J. Kim, E. Hosono, H. S. Zhou, T. Kudo, I. Honma, Nano Lett. 2008, 8, 2277-2282.

[18] R. Rurali, V. R. Coluci, D. S. Galvao, Phys. Rev. 2006, 74, 085414.

[19] R R. Bacon, J. Appl. Phys. 1960, 31, 283-290.

[20] J. L., Li, Q. S. Peng, G. Z. Bai, W. Jiang, Carbon 2005, 43, 2817 2833.

[21] H. Shioyama, T. Akita, Carbon 2003, 41, 179-198.

[22] L. M. Viculis, J. J. Mack, R. B. Kaner, Science 2003, 299, 1361.

[23] J. Zheng, H. Liu, B. Wu, Y. Guo, T. Wu, G. Yu, Y. Liu, D. Zhu, Adv Mater. 2011, 23, 2460-2463.

[24] P. Oxley, C. Brechtelsbauer, F. Ricard, N. Lewis, C. Ramshaw, Ind. Eng. Chem. Res. 2000, 39, 2175-2182.

[25] A. D. Martin, R. A. Boulos, L. J. Hubble, K. J. Hartlieb, C. L. Raston, Chem. Commun. 2011, 47, 7353-7355.

[26] K. S. Iyer, M. Norret, S. J. Dalgarno, J. L. Atwood, C. L. Raston, Angew. Chem. Int. Ed. 2008, 47, 6362-6366.

[27] N. M. Smith, B. Corry, K. S. Iyer, M. Norret, C. L. Raston, Lab Chip 2009, 9, 2021-2025.

[28] S. F. Braga, V. R. Coluci, S. B. Legoas, R. Giro, D. S. Galvao, R. H. Baughman, Nano Lett. 2004, 4, 881-884.

[29] S. Iijima, Nature 1991, 354, 56-58.

[30] O. Hod, J. Chem. Theory Comput. 2012, 8, 1360-1369.

[31] K. S. Iyer, C. L. Raston, M. Saunders, Lab Chip 2007, 7, 1121-1124.

[32] A. N. Imtani, V. K. Jindal, Materials Science, 2006, p. 11.

[33] D. H. Robertson, D. W. Brenner, J. W. Mintmire, Phys. Rev. B 1992 45, 12592-12595.

[34] O. Gülseren, T. Yildirim, S. Ciraci, Phys. Rev. B 2002, 65, 153405.

[35] K. Hashemnia, M. Farid, R. Vatankhah, Comput. Mat. Sci. 2009, 47, 79-85.

Keywords: Nano-scrolls · Shear force - Microfluidics · Graphite ·

Graphene $\cdot$ Boron nitride 


\section{Entry for the Table of Contents}

Layout 1:

Carbon and Boron Nitride Nanoscrolls

Xianjue Chen, Ramiz A. Boulos, John F. Dobson and Colin L. Raston*

$$
\text { Page - Page }
$$

Shear induced formation of carbon and boron nitride nano-scrolls

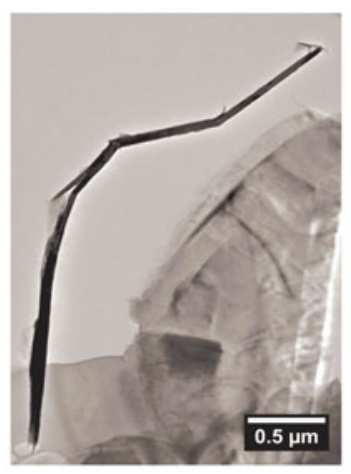

A 'top down' synthesis of carbon and hexagonal boron nitride ( $h$-BN) nanoscrolls has been developed involving the shear force within dynamic thin films on rapidly rotating surfaces in a spinning disc processor, along with a theoretical understanding of scroll formation. 


\section{Supporting Information}

\section{S1. Spinning disc processor}

Photographs of the spinning disc processor and the stainless steel disc are shown in Figure S1a and Figure S1b, respectively. Different components of the system are listed below.
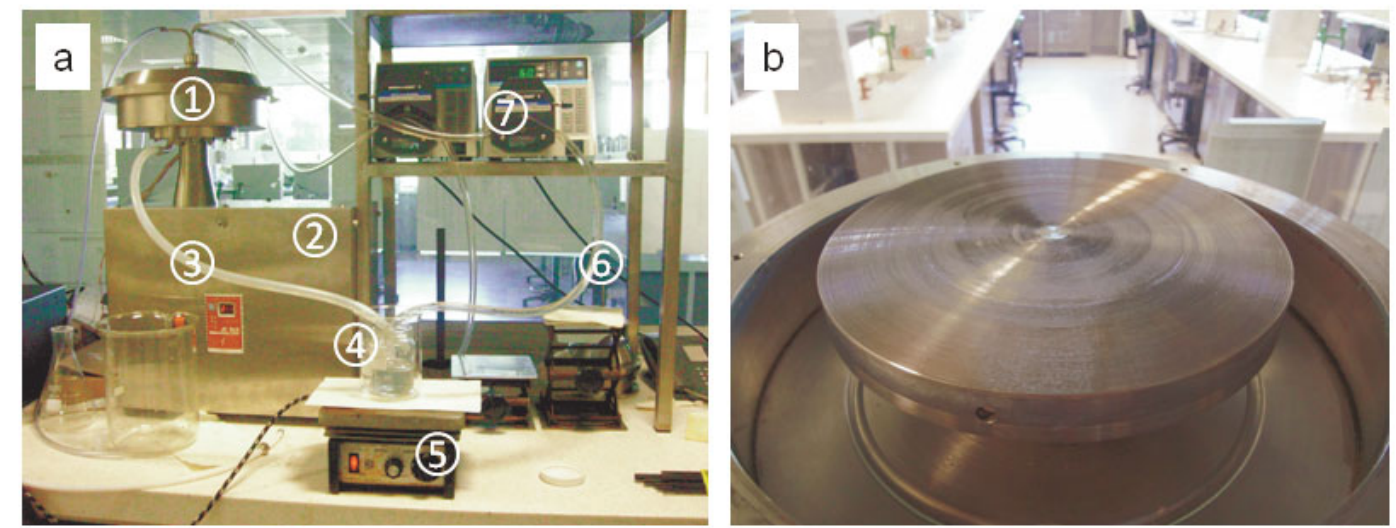

Figure S1 (a) Spinning disc processor and (b) the stainless steel disc.

\section{Components}

1. Stainless steel disc

2, Main body

3. Silicone tubes (output)

4. Suspension

5. Magnetic stirrer

6. Silicone tubes (input)

7. Flow controllers
Suspensions are fed onto the rotating disc, close to the centre, with a stainless steel housing for collecting the suspensions thrown of the edge of the disc, which are collected from and output hole.

For controlling the rotating speed and mechanical power of the disc.

For collecting and directing the outflow to the suspension reservoir.

Suspensions were temporary stored for recycling back on to the disc.

For keeping the graphite or boron nitride flakes dispersed in the solvent NMP.

For directing the suspension from the reservoir to the input of the system.

For controlling the flow rate of the suspensions in the silicone tube. 
S2. TEM images of the exfoliated BN sheets.

The BN flakes are exfoliated by the shear in the dynamic thin films using SDP. TEM images of the exfoliated BN sheets are shown in Figure S2. The exfoliation of the graphite flakes is not as effective as that of $\mathrm{BN}$, which may be due to the smaller particle size of the pristine $\mathrm{BN}$ flakes requiring less energy for exfoliation.

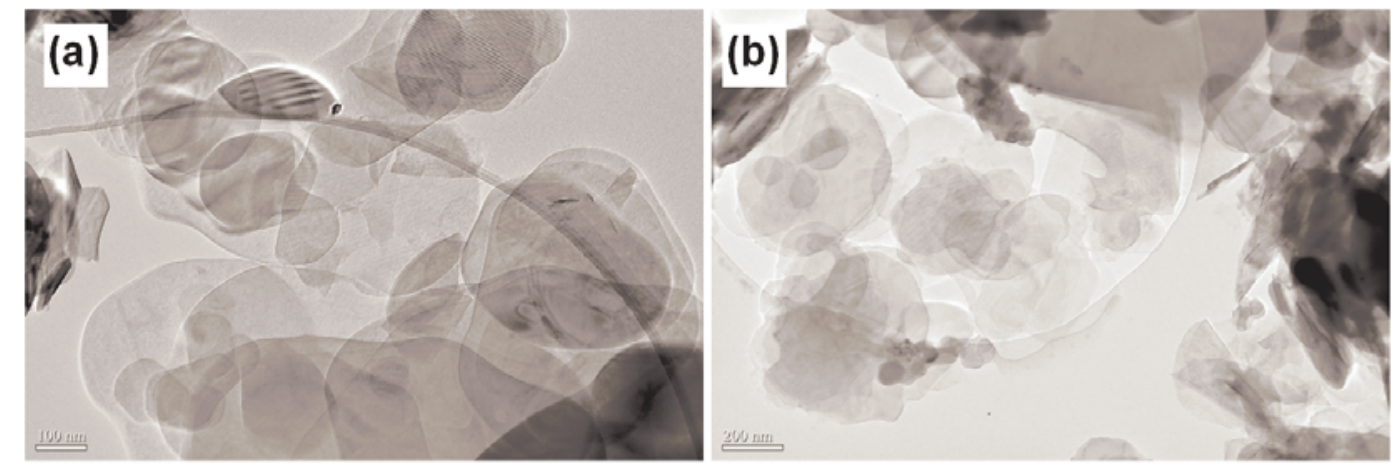

Figure S2 TEM images of the exfoliated BN sheets 


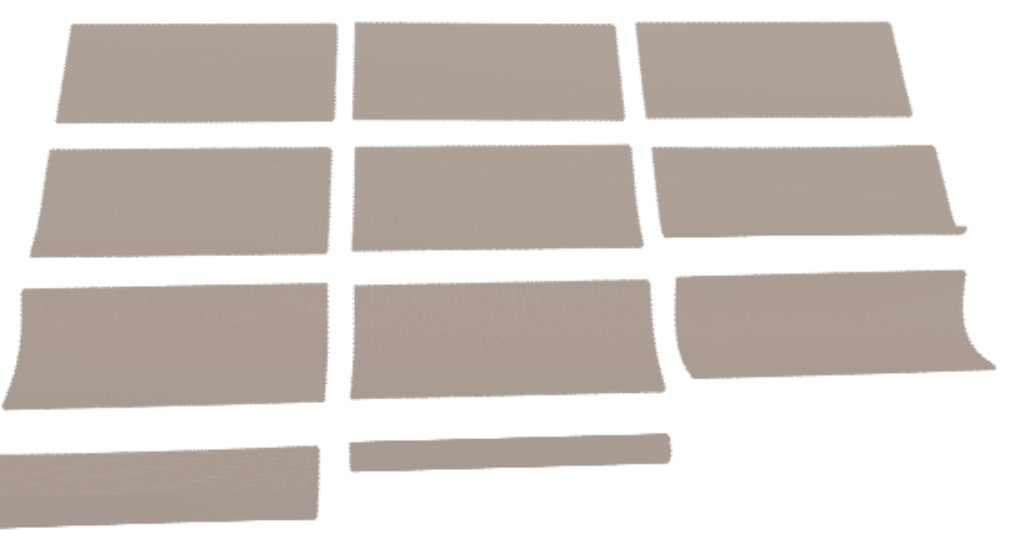

Figure S3-1 Geometry of the sheets after minimization - From top left, sheets with rollup values of $0,5,8,16,20,24,32,40,48,56$, and 60 .

Most of the minimized structures relaxed back to an almost-flat sheet with the exception of the intermediate with a rollup value of 56 and the fully scrolled sheet with a rollup value of 60 .

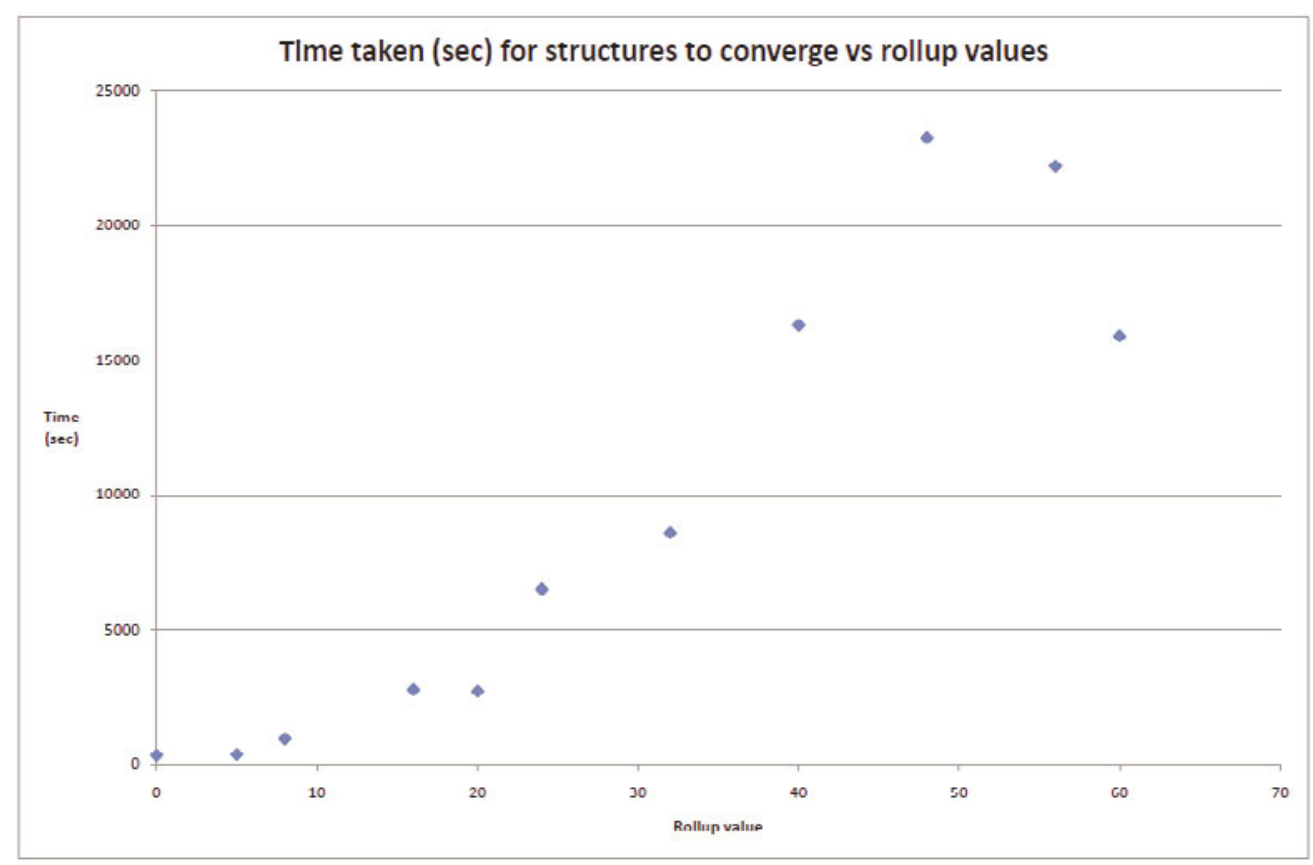

Figure S3-2 Relationship between the time take for the structures to converge and the rollup values (arctan) 